\title{
Vaginal Obstruction, CTCAE 5.0
}

National Cancer Institute

\section{Source}

National Cancer Institute. Vaginal Obstruction, CT CAE 5.0. NCI Thesaurus. Code C146696.

A disorder characterized by blockage of vaginal canal. 